\title{
Article \\ Stunting Malnutrition Associated with Severe Tooth Decay in Cambodian Toddlers
}

\author{
Eva Peris Renggli ${ }^{1, *}$, Bathsheba Turton ${ }^{2} \mathbb{D}$, Karen Sokal-Gutierrez $^{3} \mathbb{D}$, Gabriela Hondru ${ }^{4} \mathbb{D}$, Tepirou Chher ${ }^{5}$, \\ Sithan Hak ${ }^{6}$, Etienne Poirot ${ }^{7}$ and Arnaud Laillou 8
}

check for updates

Citation: Renggli, E.P.; Turton, B.; Sokal-Gutierrez, K.; Hondru, G.; Chher, T.; Hak, S.; Poirot, E.; Laillou, A. Stunting Malnutrition Associated with Severe Tooth Decay in Cambodian Toddlers. Nutrients 2021, 13, 290. https://doi.org/10.3390/ nu13020290

Academic Editor: Kirstin Vach Received: 9 December 2020

Accepted: 15 January 2021

Published: 20 January 2021

Publisher's Note: MDPI stays neutral with regard to jurisdictional claims in published maps and institutional affiliations.

Copyright: (c) 2021 by the authors. Licensee MDPI, Basel, Switzerland. This article is an open access article distributed under the terms and conditions of the Creative Commons Attribution (CC BY) license (https:// creativecommons.org/licenses/by/ $4.0 /)$.
Centre for International Health Protection, Robert Koch Institute, 13353 Berlin, Germany 2 Department of Dentistry, University Puthisistra, 12211 Phnom Penh, Cambodia; bethy.turton@gmail.com School of Public Health, University of California, Berkeley, CA 94720, USA; ksokalg@berkeley.edu UNICEF Cambodia, 12100 Phnom Penh, Cambodia; gabriela.hondru@gmail.com

5 Oral Health Bureau, Department of Preventive Medicine, Ministry of Health, 12211 Phnom Penh, Cambodia; tepirou@yahoo.com

6 Department of Preventive Medicine, Ministry of Health, 12211 Phnom Penh, Cambodia; sithan_hak@yahoo.com

7 UNICEF Chad, 1146 N’Djamena, Chad; epoirot@unicef.org

8 UNICEF Ethiopia, 1169, Addis Ababa, Ethiopia; alaillou@unicef.org

* Correspondence: evaperisrenggli@gmail.com

\begin{abstract}
Background: The persistently high prevalence of undernutrition in Cambodia, in particular stunting or chronic malnutrition, calls for innovative investigation into the risk factors that affect children's growth during critical phases of development. Methods: Secondary data analysis was performed on a subgroup of children who were present at two time points within the Cambodian Health and Nutrition Monitoring Study (CAHENMS) and who were less than 24 months of age at the nominated baseline. Data consisted of parent interviews on sociodemographic characteristics and feeding practices, and clinical measures for anthropometric measures and dental status. Logistic regression modelling was used to examine the associations between severe dental caries (tooth decay) - as indicated by the Significant Caries Index — and the presence of new cases of stunting malnutrition at follow-up. Results: There were 1595 children who met the inclusion criteria and $1307(81.9 \%)$ were followed after one year. At baseline, $14.4 \%$ of the children had severe dental caries, $25.6 \%$ presented with stunted growth. $17.6 \%$ of the children transitioned from healthy status to a low height-for-age over the observation period. Children with severe dental caries had nearly double the risk (OR = 1.8; CI 1.0-3.0) of making that transition. Conclusion: Severe caries experience was associated with poorer childhood growth and, as such, could be an underinvestigated contributor to stunting.
\end{abstract}

Keywords: severe caries; tooth decay; dental; early childhood; early childhood caries; malnutrition; undernutrition; stunting; growth and development

\section{Introduction}

Over recent decades, early childhood undernutrition has been declining globally. However, high rates of stunting have persisted, while the rates of obesity have increased [1-3]. The modern challenge of the "double burden" of undernutrition and obesity calls for an examination of the neglected risk factors that may contribute to the high prevalence of child malnutrition. Globalization and urbanization have led to a global nutrition transition, dramatically increasing young children's consumption of sugary drinks and snacks, contributing to both child obesity and tooth decay (dental caries) [4].

Dental caries is a process which leads to the destruction of tooth tissue. Dental cavities result when net demineralization occurs at the surface of the tooth in response to $\mathrm{pH}$ changes in the microbial biofilm (plaque). When the biofilm is exposed to free sugars, then acid is produced, and in the absence of protective factors such as tooth brushing 
with fluoride toothpaste, then the tooth surface will break down and carious lesions will progress [5,6]. When a carious lesion is present among children less than 6 years of age, it is commonly referred to in the literature as early childhood caries (ECC) [7]. Globally, dental caries is the most prevalent chronic disease, affecting $60-90 \%$ of schoolchildren [8]. Among the youngest age groups, most carious lesions (dental cavities) remain untreated due to limited access to dental care, particularly among low- and middle-income populations and socioeconomically disadvantaged groups [9-11]. Severe and uncontrolled dental caries can lead to oral infection and inflammation (abscesses), which can cause mouth pain, decreased appetite, inability to chew food, inadequate sleep, and chronic inflammation if persistent over time [12-14]. These factors, added to well-known aspects such as child nutrition, breastfeeding, infectious diseases, mother's health, and psychosocial stimulation, could all contribute to undernutrition [15].

In Cambodia, the current rates of both early childhood undernutrition and dental caries are among the highest in the world. Contributors include Cambodia's long period of war and humanitarian crises followed by rapid economic growth since the late 1990s, involving the introduction of ultraprocessed foods and beverages, and a dramatic increase in childhood sugar consumption $[16,17]$. Among Cambodian children under age $5,32 \%$ experience stunting and $10 \%$ wasting malnutrition [18], and the prevalence of dental caries exceeds $90 \%$ [19]. Among 3-5-year-olds, $16.1 \%$ had one or more severe, deep caries infecting the surrounding soft tissues and commonly causing mouth pain, and the prevalence of this type of dental infection increases to $86 \%$ among 6-year-old children [20-22]. In Cambodia, the high prevalence of severe caries experience by age 2, within the first 1000 days of life, elevates the concern about the potential for adverse effects on children's short-term and long-term growth and development. In fact, stunting, an indicator for chronic malnutrition, has been described to peak between 1 and 2 years of age [23]. Nevertheless, recent studies have proposed that the prevalence of stunting can increase beyond 24 months of age in suboptimal environments [24-27].

Given the high rates of chronic malnutrition and dental caries among Cambodian children, there is a pressing need to explore the potential relationship between these two conditions. This could help to better understand the role of severe dental caries as an under-recognized contributor to stunting. This longitudinal study examines the relationship between severe dental caries and anthropometric changes over a one-year period, in children under 2 years of age at baseline.

\section{Materials and Methods}

This is a secondary analysis of a longitudinal cohort study, the Cambodian Health and Nutrition Monitoring Study (CAHENMS), and an added oral health component (Figure A1). Data were collected from three Cambodian provinces: two predominantly rural northeastern provinces, Ratanakiri and Kratie; and an urban area, the capital Phnom Penh. Data were collected in 2017 (baseline) and 2018 (follow-up) using 8 trained teams specialized in conducting questionnaires, anthropometric measurements, and intraoral examinations.

The original protocol for the CAHENMS was reviewed by the National Ethics Committee for Health Research, Ministry of Health, Cambodia, prior to collecting data (117/NECHR). Written consent was obtained from the parents of the participants before the baseline data collection, and verbal consent was obtained at each subsequent contact. A data-sharing agreement was made prior to the transfer of de-identified data. This is a secondary analysis of a de-identified dataset and was considered research on nonhuman subjects. Information about the original CAHENMS study is provided in the supplement.

The study population consists of a final sub-sample of 1307 children $<24$ months of age at baseline and approximately one year older at follow-up, as presented in Figure 1. Children lost to attrition $(N=12.5 \%)$, older than 23.9 months at baseline $(N=30.5 \%)$ and with missing anthropometric measurements were not considered in the present analysis $(N=0.5 \%)$. 


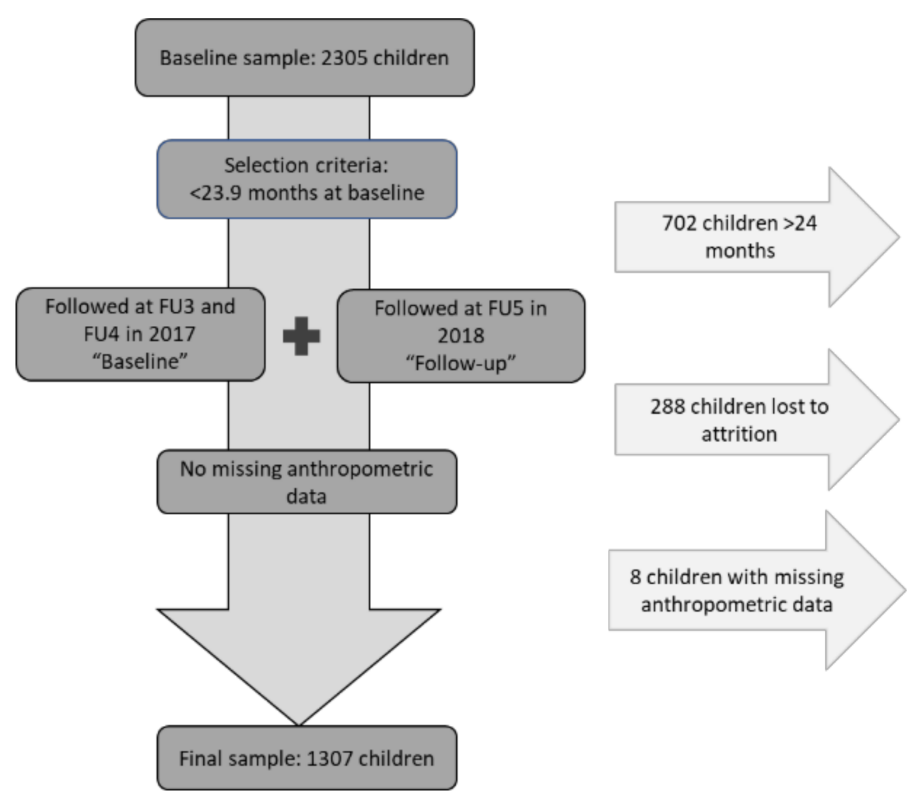

Figure 1. Flow chart depicting selection criteria of individuals included in the final sample size.

$\mathrm{G}^{*}$ power (version 3.9.1.2) was used to calculate implied power using Chi-squared tests given a sample size of 1307 participants and the intention to observe a clinically significant $5 \%$ difference in new cases of stunting (Height-for-age Z-score, $\mathrm{HAZ}<-2$ ) from 30\% down to $25 \%$. The present sample size implies a $98.6 \%$ chance of detecting a $5 \%$ difference in the incidence of stunting.

\subsection{Questionnaires}

The 2 sets of questionnaires for sociodemographic characteristics and child feeding practices were administered at baseline by trained Cambodian interviewers in Khmer, or translated into the local indigenous language for some minority populations in northeastern provinces. Dietary intake was assessed using a $24-\mathrm{h}$ recall period, based on WHO guidelines and described in published articles on child feeding practices status [28-30]. Parents/caregivers were asked to recall and report on breastfeeding frequency, consumed food groups, frequency of meals, and amount of food given during meals using as unit of measure context relevant utensils: spoons and Chan Chang Koeh, a traditional bowl in Southeast Asia.

To assess socioeconomic status (SES), questions were asked about assets, employment, and household characteristics to further calculate the wealth index using Principal Component Analysis (PCA) based on the validated tool performed by Filmer and Pritchett without expenditure data [31].

\subsection{Clinical Examinations}

Separate teams performed anthropometric measurements, and intraoral examinations and data were recorded on separate devices to ensure that examiners were blinded to the participants' status. Anthropometric measures were assessed in duplicates according to the WHO guidelines, and the mean value was further used [32]. Recumbent length was recorded in children under 2 years of age or unable to stand up, and in children above the age of 2 years height was recorded. Weight was measured with a calibrated precise scale for mother and child.

Intraoral examinations were performed by one of 8 calibrated examiners with the help of a trained assistant. All examiners achieved a kappa score of $>0.9$, indicating near-perfect agreement. The examination was done in supine position with a handheld torch and mouth mirror. The decayed, missing, and filled teeth (dmft) index as defined by WHO was used to measure dental caries experience [33]. 


\subsection{Data Analysis}

Data were delivered through Microsoft Excel and entered into IBM SPSS 25. Statistical significance was considered for $p$-values below 0.05 , and no imputation method was used. Data were cleaned and each variable was divided into relevant categories for age groups, socioeconomic status (SES), feeding practices, anthropometric measurements, and dental caries measurements. The Wealth index scores were broken down into quintiles to categorize participants by SES. Age data were based on months at baseline, and children were categorised into one of four age groups (<6 months, 6-12 months, 12-18 months, $>18$ months).

The Minimum Acceptable Diet (MAD) was calculated using the WHO Guidelines and is a composite index of breastfeeding or milk feedings, Minimum Diet Diversity and Minimum Meal Frequency; indicators being available for children aged 6 to 23.9 months at baseline. The Minimum Diet Diversity, after the 2010 guidelines, was based on whether or not the child consumed in the past $24 \mathrm{~h}$ a minimum 4 out of a list of 7 food groups. These food groups included: (1) Grains, roots and tubers; (2) Legumes and nuts; (3) Dairy products; (4) Flesh foods; (5) Eggs; (6) Vitamin A-rich fruits and vegetables; and (7) Other fruits and vegetables. The Minimum Meal Frequency was calculated on the minimum amount of times that the child received solid, semisolid or soft foods the previous day according to their age group and breastfeeding status [34,35].

As a Cambodian specific diet indicator, The Cambodian Complementary Feeding (CCF) variable was calculated based on the Nutrition Handbook for the family, prepared and adapted by the National Cambodian Nutrition Program [36]. It was based on the number of spoons of Chan Chang Koeh and determined according to age and breastfeeding status $[29,30]$.

Anthropometric measurements were converted to sex-specific height/length-for-age Z-scores using the WHO Child Growth Standards 2016 [32], and identified as stunting at $<-2$ SDs. The low proportion of obese children in the sample $(<2 \%)$, identified at $>+2$ SDs for weight-for-age, was insufficient in number to establish a comparison group. New cases of stunting were identified, i.e., children who had optimal HAZ at baseline, while at 1-year follow-up were identified with HAZ below -2 SDs. To assess caries experience, the Significant Caries Index (ScI) was calculated at baseline and follow-up by ranking individuals within age groups according to the number of teeth with carious lesions (based on the dmft index: decayed, missing or filled teeth). Subsequently, the mean of the most severe one-third of the population was calculated [37]. That value was then used to create an age-adjusted dichotomous variable to indicate the presence or absence of ScI. This index represents a more "severe" disease experience, as children with a high dmft score and presenting ScI would be more likely to develop lesions that might create mouth pain or infection, which are acknowledged consequences of carious lesions [12-14].

After cleaning and categorising the data, descriptive and multivariate analyses were performed. The Chi-squared test was used to examine differences in proportions among sociodemographic subgroups for stunting, caries, and diet indicators. Multivariate logistic regression was performed to explore the relationship between severe caries experience (by $\mathrm{ScI}$ ) at baseline and follow-up with the onset of new cases of stunting over the observation period. Gender, province, age, SES, and the MAD and CCF variables for diet indicators were included in the multivariate modelling.

\section{Results}

\subsection{Descriptive Statistics}

3.1.1. Demographic Profile and Clinical Characteristics at Baseline

Baseline participants' characteristics are shown in Table 1. Three out of four participants came from the northeastern provinces of Kratie and Ratanakiri and one quarter from Phnom Penh. There was an even gender distribution. Two-thirds of participants were 1 to 2 years of age at baseline; and over one-third of participants belonged to the lowest two wealth index quintiles. Stunted growth was present in $25.6 \%$ of the children. 
Males, children from Ratanakiri province, those from the older age groups, and those from the second quintile, "low", presented a significantly higher prevalence of stunting. Overall, $51.9 \%$ of the children presented with "any caries" at baseline and there was no statistically significant difference by gender. Those in Ratanakiri and in older age groups had a significantly higher prevalence of any caries and severe caries (by baseline ScI). When looking at SES, those in the medium quintile presented the lowest caries experience, and the lowest wealth index quintile had the higher proportion of children who had ScI at baseline compared to the other quintiles ( $21 \%$ vs. $12-14 \%, p=0.040 ; \chi^{2}$ test).

Table 1. Sociodemographic and clinical characteristics at baseline.

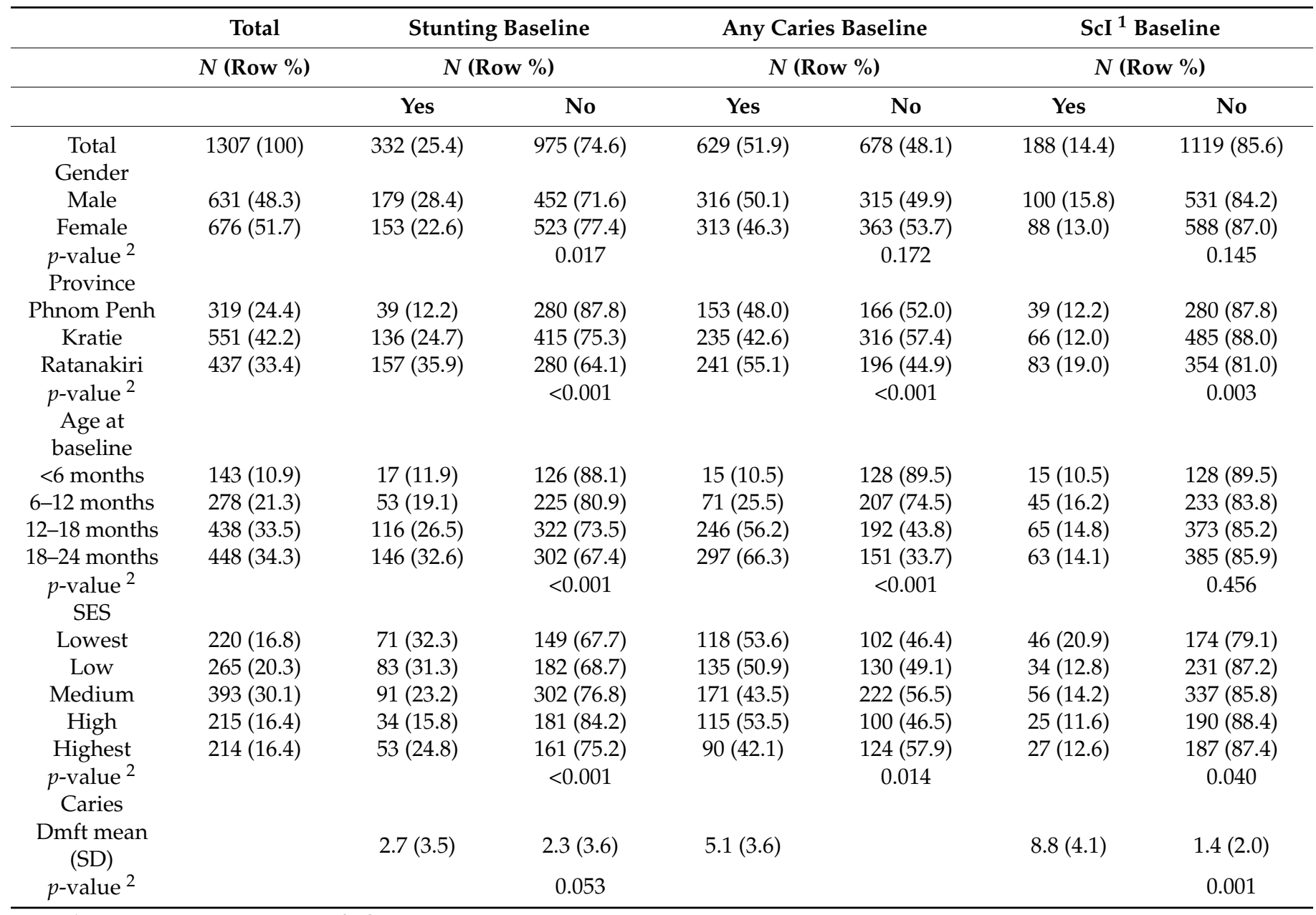

${ }^{1}$ ScI: Significant Caries Index. ${ }^{2} \chi^{2}$ test for differences among groups within the same columns or ANOVA. SES: socioeconomic status.

The mean (SD) dmft for children with caries at baseline was 5.1 (3.6). The mean cutoff of ScI by age groups at baseline was 4.7; with a mean $\mathrm{dmft}$ of 8.8 (4.1) for children with ScI and 1.4 (2.0) in children without. Children with stunting presented a dmft of 2.7 (3.5) and children without stunting $2.3(3.6)(p=0.053)$.

\subsubsection{Clinical Characteristics at Follow-Up}

The participants' characteristics at follow-up are shown in Table 2. Stunted growth was present in $39.9 \%$ of the children. In contrast to baseline, females had a significantly higher chance of presenting with stunting at follow-up ( $42.2 \%$ vs. $36.1 \%, p=0.026 \chi^{2}$ test). Children from Ratanakiri province, approximately $>12$ months of age, and from the "low" SES quintile, continued to present the highest prevalence of stunting. 
Table 2. Sociodemographic and clinical characteristics at follow-up.

\begin{tabular}{|c|c|c|c|c|c|c|}
\hline & \multicolumn{2}{|c|}{ Stunting Follow-Up } & \multicolumn{2}{|c|}{ Any Caries Follow-Up } & \multicolumn{2}{|c|}{ Sci ${ }^{1}$ Follow-Up } \\
\hline & \multicolumn{2}{|c|}{$N($ Row \%) } & \multicolumn{2}{|c|}{$N($ Row \%) } & \multicolumn{2}{|c|}{$N($ Row \%) } \\
\hline & Yes & No & Yes & No & Yes & No \\
\hline $\begin{array}{l}\text { Total } \\
\text { Gender }\end{array}$ & $513(39.3)$ & 794 (60.7) & $831(63.6)$ & $476(36.4)$ & $193(14.8)$ & $1114(85.2)$ \\
\hline Male (631) & $228(36.1)$ & $403(63.9)$ & $401(63.5)$ & $230(36.5)$ & 111 (17.6) & $520(82.4)$ \\
\hline $\begin{array}{c}\text { Female }(676) \\
p \text {-value } \\
\text { Province }\end{array}$ & $285(42.2)$ & $\begin{array}{c}391(57.8) \\
0.026\end{array}$ & 430 (63.6) & $\begin{array}{c}246(36.4) \\
0.982\end{array}$ & $82(12.1)$ & $\begin{array}{c}594(87.9) \\
0.005\end{array}$ \\
\hline $\begin{array}{l}\text { Phnom Penh } \\
\text { (319) }\end{array}$ & $74(23.2)$ & $245(76.8)$ & 255 (79.9) & $64(20.1)$ & $60(18.8)$ & 259 (81.2) \\
\hline Kratie (551) & $206(37.4)$ & 345 (62.6) & 318 (57.7) & $233(42.3)$ & 79 (14.3) & $472(85.7)$ \\
\hline $\begin{array}{c}\text { Ratanakiri (437) } \\
p \text {-value } \\
3\end{array}$ & $233(53.3)$ & $\begin{array}{c}204(46.7) \\
<0.001\end{array}$ & 258 (59.9) & $\begin{array}{c}179(41.0) \\
<0.001\end{array}$ & $54(12.4)$ & $\begin{array}{c}383(87.6) \\
0.044\end{array}$ \\
\hline $\begin{array}{l}\text { Approx. age } \\
\text { follow-up }{ }^{2}\end{array}$ & & & & & & \\
\hline $\begin{array}{l}<12 \text { months } \\
\text { (143) }\end{array}$ & $74(51.7)$ & $69(48.3)$ & $52(36.4)$ & $91(63.6)$ & $32(22.4)$ & 111 (77.6) \\
\hline $\begin{array}{l}\text { 12-18 months } \\
\text { (278) }\end{array}$ & $109(39.2)$ & $169(60.8)$ & $148(53.2)$ & $130(46.8)$ & $15(5.4)$ & 263 (94.6) \\
\hline $\begin{array}{l}\text { 18-24 months } \\
\text { (438) }\end{array}$ & $160(36.5)$ & $278(63.5)$ & $283(64.6)$ & $155(35.4)$ & 68 (15.5) & $370(84.5)$ \\
\hline $\begin{array}{l}\text { 24-36 months } \\
\text { (448) }\end{array}$ & $170(37.9)$ & $278(62.1)$ & 348 (77.7) & $100(22.3)$ & $78(17.4)$ & $370(82.6)$ \\
\hline $\begin{array}{c}p \text {-value } \\
\text { SES }\end{array}$ & & 0.011 & & $<0.001$ & & $<0.001$ \\
\hline Lowest (220) & $99(45.0)$ & $121(55.0)$ & $107(48.6)$ & $113(51.4)$ & $21(9.5)$ & 199 (90.5) \\
\hline Low (265) & 147 (55.5) & $118(44.5)$ & $168(63.4)$ & $97(36.6)$ & $37(14.0)$ & $228(86.0)$ \\
\hline Medium (393) & $130(33.1)$ & $263(66.9)$ & $259(65.9)$ & $134(34.1)$ & $67(17.0)$ & $326(83.0)$ \\
\hline High (215) & $58(27.0)$ & $157(73.0)$ & $136(63.3)$ & 79 (36.7) & $34(15.8)$ & $181(84.2)$ \\
\hline $\begin{array}{c}\text { Highest (214) } \\
p \text {-value } \\
\text { Caries }\end{array}$ & 79 (36.9) & $\begin{array}{c}135(63.1) \\
<0.001\end{array}$ & $161(75.2)$ & $\begin{array}{c}53(24.8) \\
<0.001\end{array}$ & $34(15.9)$ & $\begin{array}{c}180(84.1) \\
0.140\end{array}$ \\
\hline $\begin{array}{l}\text { Dmft mean } \\
\text { (SD) }\end{array}$ & $3.2(3.9)$ & $3.3(4.1)$ & $5.1(3.9)$ & & $10.6(4.0)$ & $2.0(2.2)$ \\
\hline$p$-value ${ }^{3}$ & & 0.800 & & & & $<0.001$ \\
\hline
\end{tabular}

${ }^{1}$ ScI: Significant Caries Index. ${ }^{2}$ Approximate age mean (SD) at follow-up: 30.79 (6.52). ${ }^{3} \chi^{2}$ test for differences among groups within the same columns or ANOVA.

Overall, $63.6 \%$ of the children at follow-up presented dental caries. A higher proportion of males than females presented with ScI at follow-up ( 17.6 vs. $12.1 \%, p=0.005 ; \chi^{2}$ test). Children living in Phnom Penh experienced a greater increase in caries and demonstrated higher overall caries experience at follow-up compared to other provinces. Differences in caries experience were seen among the five SES groups, whereby the highest SES group had a greater proportion of children with any caries (75\% vs. $49-66 \%)$ and higher mean $\operatorname{dmft}\left(p<0.001 ; \chi^{2}\right.$ test, ANOVA). A higher prevalence of ScI among the lowest SES was no longer significant at follow-up.

The mean (SD) dmft for children with caries at follow-up was 5.1 (3.9). The mean cutoff of ScI by age groups at follow-up was 7.2, with a mean dmft of 10.6 (4.0) for children with ScI and 2.0 (2.2) in children without. The mean dmft in stunted children was 3.2 (3.9) and in non-stunted children $3.3(4.1)(p=0.800)$.

3.1.3. New Cases of Stunting, Significant Caries, and Dietary Adequacy by Sociodemographic Characteristics

Overall, $17.6 \%$ of children transitioned from normal values at baseline to suboptimal level of length/height-for-age at follow-up. Children who presented stunted malnutrition 
at baseline were excluded from the analysis $(n=332)$. Females were significantly more likely to transition to a suboptimal level of height-for-age at follow-up; one out of four females and one out of five males presented new cases of stunting $\left(p=0.001 ; \chi^{2}\right.$ test). Children in Ratanakiri had a higher proportion of new cases of stunting when compared to other provinces ( $p$-value $\leq 0.001 ; \chi^{2}$ test). Children from the youngest age group, at approximately 18 months at follow-up, presented the highest frequency of new cases of stunting ( $p$-value $<0.001, \chi^{2}$ test). Regarding SES, the children from the second wealth index quintile (low) presented the highest prevalence of new cases of stunted malnutrition $\left(p\right.$-value $\leq 0.001 ; \chi^{2}$ test $)$.

Measures of dietary adequacy by sociodemographic characteristics are presented in Table 3. Males were more likely than females to have adequate dietary intake, including a significantly higher likelihood of achieving CCF $\left(62.6 \%\right.$ vs. $51.3 \%, p<0.001 ; \chi^{2}$ test). Phnom Penh had the highest proportion, and Ratanakiri the lowest proportion of children who met the criteria for MAD and CCF. Children 0-6 months of age presented the highest proportion of acceptable CCF intake $\left(p<0.001 ; \chi^{2}\right.$ test). Children 12 months and over were more likely than children age 6-12 months to have received MAD $\left(p<0.001 ; \chi^{2}\right.$ test). Children in the first 4 SES quintiles demonstrated a progressively higher chance of meeting dietary criteria by MAD and CCF; however, those in the highest quintile appeared to have a lower dietary quality than those in the high quintile $\left(p<0.001 ; \chi^{2}\right.$ test).

Table 3. Sociodemographic characteristics of new cases of stunting, severe caries and dietary adequacy.

\begin{tabular}{|c|c|c|c|c|c|c|c|c|c|c|}
\hline & \multirow{2}{*}{\multicolumn{2}{|c|}{$\begin{array}{c}\begin{array}{c}\text { New Cases } \\
\text { Stunting }\end{array} \\
\text { Valid: } 975 \\
\text { Missing: } 332 \\
N \text { (Row \%) }\end{array}$}} & \multicolumn{2}{|c|}{ ScI Baseline } & \multicolumn{2}{|c|}{ ScI Follow-Up } & \multicolumn{2}{|c|}{$\mathrm{CCF}^{1}$} & \multicolumn{2}{|c|}{ MAD $^{2}$} \\
\hline & & & \multicolumn{2}{|c|}{$\begin{array}{l}\text { Valid: } 1307 \\
\text { Missing: } 0 \\
N(\text { Row \%) }\end{array}$} & \multicolumn{2}{|c|}{$\begin{array}{l}\text { Valid: } 1307 \\
\text { Missing: } 0 \\
N \text { (Row \%) }\end{array}$} & \multicolumn{2}{|c|}{$\begin{array}{c}\text { Valid: } 1127 \\
\text { Missing: } 180 \\
N \text { (Row \%) }\end{array}$} & \multicolumn{2}{|c|}{$\begin{array}{c}\text { Valid: } 1017 \\
\text { Missing: } 290 \\
N \text { (Row \%) }\end{array}$} \\
\hline & Yes & No & Yes & No & Yes & No & Yes & No & Yes & No \\
\hline Total & $\begin{array}{c}230 \\
(17.6)\end{array}$ & $\begin{array}{c}745 \\
(57.0)\end{array}$ & $\begin{array}{c}188 \\
(14.4)\end{array}$ & $\begin{array}{c}1119 \\
(85.6)\end{array}$ & $\begin{array}{c}193 \\
(14.8)\end{array}$ & $\begin{array}{c}1114 \\
(85.2)\end{array}$ & $\begin{array}{c}201 \\
(15.4)\end{array}$ & $\begin{array}{c}816 \\
(62.4)\end{array}$ & $\begin{array}{c}640 \\
(49.0)\end{array}$ & $\begin{array}{c}487 \\
(37.3)\end{array}$ \\
\hline $\begin{array}{c}\text { Gender } \\
\text { Male (631) }\end{array}$ & $85(18.8)$ & $\begin{array}{c}367 \\
(81.2)\end{array}$ & $100(15.8)$ & $\begin{array}{c}531 \\
(84.2)\end{array}$ & $\begin{array}{c}111 \\
(17.6)\end{array}$ & $\begin{array}{c}520 \\
(82.4)\end{array}$ & $\begin{array}{c}113 \\
(22.1)\end{array}$ & $\begin{array}{c}398 \\
(77.9)\end{array}$ & $\begin{array}{c}343 \\
(62.6)\end{array}$ & $\begin{array}{c}205 \\
(37.4)\end{array}$ \\
\hline $\begin{array}{c}\text { Female (676) } \\
p \text {-value } \\
\text { Province }\end{array}$ & $\begin{array}{c}145 \\
(27.7)\end{array}$ & $\begin{array}{l}378 \\
(72.3) \\
0.001\end{array}$ & 88 (13.0) & $\begin{array}{c}588 \\
(87.0) \\
0.145\end{array}$ & $82(12.1)$ & $\begin{array}{l}594 \\
(87.9) \\
0.005\end{array}$ & 88 (17.4) & $\begin{array}{c}418 \\
(82.6) \\
0.059\end{array}$ & $\begin{array}{c}297 \\
(51.3)\end{array}$ & $\begin{array}{c}282 \\
(48.7) \\
<0.001\end{array}$ \\
\hline $\begin{array}{c}\text { Phnom Penh } \\
\text { (319) }\end{array}$ & $42(15.0)$ & $\begin{array}{c}238 \\
(85.0)\end{array}$ & $39(12.2)$ & $\begin{array}{c}280 \\
(87.8)\end{array}$ & $60(18.8)$ & $\begin{array}{c}259 \\
(81.2)\end{array}$ & $94(38.5)$ & $\begin{array}{c}150 \\
(61.5)\end{array}$ & $\begin{array}{c}201 \\
(71.3)\end{array}$ & $81(28.7)$ \\
\hline Kratie (551) & $97(23.4)$ & $\begin{array}{c}317 \\
(76.6)\end{array}$ & $66(12.0)$ & $\begin{array}{c}485 \\
(88.0)\end{array}$ & 79 (14.3) & $\begin{array}{c}472 \\
(85.7)\end{array}$ & 77 (17.9) & $\begin{array}{c}357 \\
(82.1)\end{array}$ & $\begin{array}{c}257 \\
(53.3)\end{array}$ & $\begin{array}{c}225 \\
(46.7)\end{array}$ \\
\hline $\begin{array}{c}\text { Ratanakiri } \\
(437) \\
p \text {-value } \\
\end{array}$ & $91(32.5)$ & $\begin{array}{c}189 \\
(67.5) \\
<0.001\end{array}$ & $83(19.0)$ & $\begin{array}{c}354 \\
(81.0) \\
0.003\end{array}$ & $54(12.4)$ & $\begin{array}{c}383 \\
(87.6) \\
0.044\end{array}$ & $30(8.7)$ & $\begin{array}{c}313 \\
(91.3) \\
<0.001\end{array}$ & $\begin{array}{c}182 \\
(50.1)\end{array}$ & $\begin{array}{c}181 \\
(49.9) \\
<0.001\end{array}$ \\
\hline $\begin{array}{l}\text { Approx. age } \\
\text { follow-up }\end{array}$ & & & & & & & & & & \\
\hline $\begin{array}{c}<12 \text { months } \\
\text { (143) }\end{array}$ & $58(46.0)$ & $68(54.0)$ & 15 (10.5) & $\begin{array}{c}128 \\
(89.5)\end{array}$ & $32(22.4)$ & $\begin{array}{c}111 \\
(77.6)\end{array}$ & / & / & $\begin{array}{c}106 \\
(89.1)\end{array}$ & $13(10.9)$ \\
\hline $\begin{array}{c}12-18 \\
\text { months } \\
(278)\end{array}$ & $63(28.0)$ & $\begin{array}{c}162 \\
(72.0)\end{array}$ & 45 (16.2) & $\begin{array}{c}233 \\
(83.8)\end{array}$ & $15(5.4)$ & $\begin{array}{c}263 \\
(94.6)\end{array}$ & $28(10.9)$ & $\begin{array}{c}228 \\
(89.1)\end{array}$ & $\begin{array}{c}122 \\
(67.8)\end{array}$ & $58(32.2)$ \\
\hline
\end{tabular}


Table 3. Cont.

\begin{tabular}{|c|c|c|c|c|c|c|c|c|c|c|}
\hline & \multirow{2}{*}{\multicolumn{2}{|c|}{$\begin{array}{c}\begin{array}{c}\text { New Cases } \\
\text { Stunting }\end{array} \\
\text { Valid: } 975 \\
\text { Missing: } 332 \\
\text { N (Row \%) }\end{array}$}} & \multirow{2}{*}{\multicolumn{2}{|c|}{$\begin{array}{l}\text { ScI Baseline } \\
\text { Valid: } 1307 \\
\text { Missing: } 0 \\
N \text { (Row \%) }\end{array}$}} & \multirow{2}{*}{\multicolumn{2}{|c|}{$\begin{array}{c}\text { ScI Follow-Up } \\
\text { Valid: } 1307 \\
\text { Missing: } 0 \\
N \text { (Row \%) }\end{array}$}} & \multirow{2}{*}{\multicolumn{2}{|c|}{$\begin{array}{c}\text { CCF }^{1} \\
\text { Valid: } 1127 \\
\text { Missing: } 180 \\
N(\text { Row \%) }\end{array}$}} & \multirow{2}{*}{\multicolumn{2}{|c|}{$\begin{array}{c}\text { MAD }^{2} \\
\text { Valid: } 1017 \\
\text { Missing: } 290 \\
\text { N (Row \%) }\end{array}$}} \\
\hline & & & & & & & & & & \\
\hline & Yes & No & Yes & No & Yes & No & Yes & No & Yes & No \\
\hline $\begin{array}{c}18-24 \\
\text { months } \\
(438)\end{array}$ & $55(17.1)$ & $\begin{array}{c}267 \\
(82.9)\end{array}$ & $65(14.8)$ & $\begin{array}{c}373 \\
(85.2)\end{array}$ & $68(15.5)$ & $\begin{array}{c}370 \\
(84.5)\end{array}$ & $92(23.3)$ & $\begin{array}{c}303 \\
(76.7)\end{array}$ & $\begin{array}{c}167 \\
(40.0)\end{array}$ & $\begin{array}{c}251 \\
(60.0)\end{array}$ \\
\hline $\begin{array}{c}24-36 \\
\text { months } \\
(448)\end{array}$ & $54(17.9)$ & $\begin{array}{c}248 \\
(82.1)\end{array}$ & $63(14.1)$ & $\begin{array}{c}385 \\
(85.9)\end{array}$ & 78 (17.4) & $\begin{array}{c}370 \\
(82.6)\end{array}$ & $81(22.1)$ & $\begin{array}{c}225 \\
(77.9)\end{array}$ & $\begin{array}{c}245 \\
(59.8)\end{array}$ & $\begin{array}{c}165 \\
(40.2)\end{array}$ \\
\hline $\begin{array}{c}p \text {-value } \\
\text { SES }\end{array}$ & & $<0.001$ & & 0.456 & & $<0.001$ & & $<0.001$ & & $<0.001$ \\
\hline Lowest (220) & 35 (23.5) & $\begin{array}{c}114 \\
(76.5)\end{array}$ & $46(20.9)$ & $\begin{array}{c}174 \\
(79.1)\end{array}$ & $21(9.5)$ & $\begin{array}{c}199 \\
(90.5)\end{array}$ & $23(12.3)$ & $\begin{array}{c}164 \\
(87.7)\end{array}$ & 79 (43.6) & $\begin{array}{c}102 \\
(56.4)\end{array}$ \\
\hline Low (265) & 77 (42.3) & $\begin{array}{c}105 \\
(57.5)\end{array}$ & 34 (12.8) & $231(7.2)$ & 37 (14.0) & $\begin{array}{c}228 \\
(86.0)\end{array}$ & $22(11.2)$ & $\begin{array}{c}175 \\
(88.8)\end{array}$ & $\begin{array}{c}139 \\
(61.2)\end{array}$ & $88(38.8)$ \\
\hline $\begin{array}{l}\text { Medium } \\
\text { (393) }\end{array}$ & 57 (18.9) & $\begin{array}{c}245 \\
(81.1)\end{array}$ & $56(14.2)$ & $\begin{array}{c}337 \\
(85.8)\end{array}$ & $67(17.0)$ & $\begin{array}{c}326 \\
(83.0)\end{array}$ & $57(20.0)$ & $\begin{array}{c}228 \\
(80.0)\end{array}$ & $\begin{array}{c}196 \\
(59.9)\end{array}$ & $\begin{array}{c}131 \\
(40.1)\end{array}$ \\
\hline High (215) & $28(15.5)$ & $\begin{array}{c}153 \\
(84.5)\end{array}$ & 25 (11.6) & $\begin{array}{c}190 \\
(88.4)\end{array}$ & $34(15.8)$ & $\begin{array}{c}181 \\
(84.2)\end{array}$ & $60(34.9)$ & $\begin{array}{c}112 \\
(65.1)\end{array}$ & $\begin{array}{c}139 \\
(69.5)\end{array}$ & $61(30.5)$ \\
\hline $\begin{array}{l}\text { Highest } \\
(214)\end{array}$ & $33(20.5)$ & $\begin{array}{c}128 \\
(79.5)\end{array}$ & 27 (12.6) & $\begin{array}{c}187 \\
(87.4)\end{array}$ & 34 (15.9) & $\begin{array}{c}180 \\
(84.1)\end{array}$ & $39(22.2)$ & $\begin{array}{c}137 \\
(77.8)\end{array}$ & 87 (45.3) & $\begin{array}{c}105 \\
(54.7)\end{array}$ \\
\hline$p$-value ${ }^{3}$ & & $<0.001$ & & 0.040 & & 0.140 & & $<0.001$ & & $<0.001$ \\
\hline
\end{tabular}

${ }^{1}$ CCF: Cambodian complementary feeding. ${ }^{2}$ MAD: Minimum acceptable diet. ${ }^{3} \chi^{2}$ test for differences among groups within the same columns or ANOVA.

\subsection{Multivariate Analysis: Logistic Regression}

Results of multivariate logistic regression on odds ratios for new cases of stunting based on severe dental caries by ScI at baseline and follow-up are presented in Table 4 (Models 1 and 2).

Table 4. Logistic regression models for the risk of stunting by caries experience, sociodemographic and diet indicators.

New Cases Stunting

\begin{tabular}{|c|c|c|c|c|}
\hline & \multicolumn{2}{|c|}{ Based on ScI at Baseline MODEL 1} & \multicolumn{2}{|c|}{ Based on ScI at Follow-Up MODEL 2} \\
\hline & OR $(95 \% \mathrm{CI})$ & $p$-Value & OR $(95 \%$ CI) & $p$-Value \\
\hline \multicolumn{5}{|l|}{ Caries experience } \\
\hline $\begin{array}{l}\text { Had ScI } \\
\text { Gender } \\
\text { Male }^{1}\end{array}$ & $0.8(0.5-1.5)$ & 0.524 & $1.8(1.0-3.0)$ & 0.039 \\
\hline $\begin{array}{c}\text { Female } \\
\text { Province }\end{array}$ & $1.7(1.1-2.5)$ & 0.012 & $1.7(1.2-2.6)$ & 0.009 \\
\hline \multicolumn{5}{|l|}{ Phnom Penh ${ }^{1}$} \\
\hline Kratie & $2.3(1.3-4.0)$ & 0.003 & $2.5(1.4-4.3)$ & 0.002 \\
\hline Ratanakiri & $3.1(1.7-5.6)$ & $<0.001$ & $3.3(1.8-6.0)$ & $<0.001$ \\
\hline \multicolumn{5}{|l|}{ Age at baseline } \\
\hline $12-18$ months & $0.5(0.3-0.8)$ & 0.009 & $0.4(0.3-0.8)$ & 0.004 \\
\hline $\begin{array}{l}\text { 18-24 months } \\
\text { SES }\end{array}$ & $0.6(0.4-1.0)$ & 0.068 & $0.5(0.3-0.9)$ & 0.027 \\
\hline
\end{tabular}


Table 4. Cont.

\begin{tabular}{|c|c|c|c|c|}
\hline & \multicolumn{4}{|c|}{ New Cases Stunting } \\
\hline & \multicolumn{2}{|c|}{ Based on ScI at Baseline MODEL 1} & \multicolumn{2}{|c|}{ Based on ScI at Follow-Up MODEL 2} \\
\hline & OR $(95 \% \mathrm{CI})$ & $p$-Value & OR $(95 \% \mathrm{CI})$ & $p$-Value \\
\hline \multicolumn{5}{|l|}{ Lowest ${ }^{1}$} \\
\hline Low & $2.0(1.0-3.7)$ & 0.040 & $2.0(1.0-3.8)$ & 0.038 \\
\hline Medium & $0.7(0.3-1.3)$ & 0.211 & $0.7(0.3-1.2)$ & 0.195 \\
\hline High & $1.0(0.5-2.0)$ & 0.964 & $1.0(0.5-2.0)$ & 0.978 \\
\hline Highest & $1.3(0.6-2.5)$ & 0.512 & $1.3(0.7-2.6)$ & 0.422 \\
\hline \multicolumn{5}{|c|}{ Diet indicators } \\
\hline \multicolumn{5}{|c|}{ No CCF ${ }^{1}$} \\
\hline CCF & $1.2(0.8-1.9)$ & 0.333 & $1.2(0.8-1.9)$ & 0.367 \\
\hline \multicolumn{5}{|l|}{ No MAD ${ }^{1}$} \\
\hline MAD & $1.9(1.2-3.2)$ & 0.011 & $1.9(1.1-3.1)$ & 0.014 \\
\hline
\end{tabular}

Having severe caries by ScI at baseline was not associated with developing new-onset stunting, although children with ScI at follow-up had approximately twice the risk with an odds ratio of $1.8\left(95 \% \mathrm{CI}=1.0-3.0, p=0.039 ; \chi^{2}\right.$ test $)$ after controlling for gender, province, age, SES, and diet indicators. The highest risk for developing new cases of stunting was seen in females (OR 1.7; CI 1.2, 2.6) from rural areas (OR 2.5-3.3; CI 1.4, 6.0), at the age of approximately $1-2$ years at follow-up ( $<12$ months at baseline) (OR 0.4-0.5; CI $0.3,0.9$ ), and coming from the second lowest SES quintile (OR 2.0; CI 1.0, 3.8), even in the presence of MAD (OR 1.9; CI 1.1, 3.1).

Collinearity between MAD and CCF is taken into consideration and ruled out because both variables capture different aspects of the feeding practices.

\section{Discussion}

This secondary analysis of longitudinal data on Cambodian children under age 2 at baseline and 1 year later at follow-up showed that children with severe dental caries (as indicated by the ScI) had almost twice the odds of developing chronic malnutrition. This suggests that dental caries experience could be an important contributor to child growth restriction at a critical stage of child development. The results of the present analysis align with some cross-sectional and longitudinal studies that reported a positive association between severe dental caries and different forms of undernutrition [38-41]. The present study is unique in that it uses a longitudinal study design, controlled for the children's dietary intake, and included children under 2 years of age at baseline from a population with severe caries experience.

A number of systematic reviews have examined the relationship between dental caries and malnutrition; some studies show association of caries with undernutrition, others with obesity, and others show no association [42-47]. The differences in child age, country's socioeconomic standing, and socioeconomic and behavioural disparities within the countries may explain the disparate findings in the literature. Moreover, the caries-malnutrition relationship appears to play out differently in urban and rural areas, different SES groups, and different countries and regions based on their stage in the nutrition transition [48-52]. The data from this study suggested that children in predominantly rural northeastern provinces had worse baseline dietary, anthropometric, and caries experience. However, children in the predominantly urban Phnom Penh presented with worse caries experience over the observation period, which may be explained by higher sugar consumption.

Suboptimal dietary, anthropometric, and caries indicators were seen in the low/lowest SES population; however, the highest SES population did not present the best outcomesthe wealthiest SES group demonstrated lower rates of dietary adequacy and higher rates of dental caries and undernutrition compared to the adjacent lower SES strata. Furthermore, some children in the higher SES strata may be vulnerable to overnutrition [53], but the 
limited number of obese participants in the sample precluded analysis of the relationship of severe dental caries and overnutrition. There is consensus that frequent consumption of high-calorie and sugary foods and drinks contributes to dental caries and overweight $[6,54]$. On the other hand, consuming sugary foods and drinks may also displace the intake of nutritious foods and drinks, and contribute to dental caries and undernutrition $[55,56]$. This suggests that, in low-middle-income countries, there may be a "u-shaped" association between SES and dietary adequacy, caries and nutritional status, rather than the linear relationship between SES and good nutrition and oral health generally described in higherincome countries $[53,57,58]$. Nonetheless, there is a paucity of research about the role of sugary diets in undernutrition, including the comorbidity with dental caries. This may be explained by the lack of oral health awareness among non-dental medical and public health professionals [59], and the economic power of the food and beverage companies that sell sugary snacks and beverages as well as oral health care products, and sponsor caries research that focuses on dental care products rather than on the cause of caries: sugar [60].

In addition, a growing body of research highlights gender inequalities in child nutrition and health $[61,62]$. In this sample, females showed worse diet and anthropometric outcomes, and males presented more severe caries experience at follow-up. However, Cambodian national data show no substantial difference in nutritional outcomes by gender in children under $5[18,63]$. The findings also support the understanding that both dental caries and chronic undernutrition are socially driven phenomena with complex and poorly studied interrelationships with each other and with sociocultural, economic and behavioural factors. In this study sample, it appears that male infants/toddlers, and those in the higher SES strata may have consumed larger quantities of food as implied by the CCF data, which may protect first against growth faltering; however, the same subgroups may also have greater consumption of sugary foods and drinks, thereby contributing to the finding of more severe caries experience, and associated long-term growth faltering. This highlights the need to address the structural drivers of early childhood caries and undernutrition from early infancy onward to reduce the long-term adverse impacts of dental caries on children's nutrition, health, and development [13].

Several limitations should be recognized in this study. The CAHENMS and the oral health survey were not specifically intended to examine the impact of tooth decay on malnutrition, and lacked detailed data on maternal health, education, and comorbidities that might contribute to the higher risk for stunting in the presence of severe caries. The dietary surveys were subject to recall bias and the diet indicators of this secondary analysis presented some constraints. The WHO indicator, Minimal Acceptable Diet (MAD) is not country-specific, and many Cambodian food items were missed. Moreover, the Minimal Diet Diversity (MDD) does not include breast milk as it has been recently proposed in the most recent guidelines. The Cambodian Complementary Feeding (CCF) indicator includes the quantity of complementary feeding and breastfeeding but not the frequency of meals per day. Thereby, the diet indicators may not have fully captured children's diet in Cambodia and data were not collected on daily child consumption of non-nutritious food and drinks (junk food). Those food groups contain high amounts of sugar, and are hypothesized to be primary causes of caries and associated stunted growth. Finally, as malnutrition and dental caries are multifactorial diseases that evolve over time [64], this one-year follow-up does not capture the cumulative impact of disease, the mitigating effect of oral hygiene and dental treatment, and a longer follow-up considering these factors is warranted. This would also allow for change modelling of trends in growth and development for which binary logistic regression may not be sensitive.

In order to use human and financial resources wisely, it is important to work closely with programmes focused on the prevention of non-communicable diseases (NCDs) that share common risk factors with tooth decay [65]. Although many countries have introduced school-based caries-prevention programmes with successful outcomes [66,67], most caries risk factors and adverse outcomes begin in early childhood, before the child attends school and before the child sees a dentist. Thus, there is an evidence-based opportunity to integrate 
caries prevention into existing primary care maternal-child health programmes from birth onwards [68].

The findings of this study suggest that reducing the severity of dental caries could, in turn, reduce the long-term adverse impact on linear growth among young children. Therefore, it could be justified to incorporate caries prevention initiatives with general child nutrition and health promotion initiatives. Such interventions might include supporting adequate infant breast- and complementary feeding $[69,70]$, babyWASH and consumption of safe water [71], and healthy environments with restricted access to sugar [72]. This should go along with an improvement of oral hygiene practices-toothbrushing two times a day with fluoride toothpaste [73] — and oral health initiatives such as population-based fluoride [74,75], and preventing caries lesions through application of sealants [76], fluoride varnishes [73,77], and topical silver and fluoride agents [78]. Finally, particularly relevant to the Cambodian context of widespread food insecurity is the need for political will to limit the global marketing of low-cost, ultra-processed junk food and sugar-sweetened drinks to improve child nutritional status, decrease the incidence of dental caries, and ultimately support children's growth and development.

\section{Conclusions}

This is the first longitudinal study to examine the association between dental caries and stunted growth in children under 2 years in Cambodia, and to demonstrate that severe tooth decay is associated with developing stunting malnutrition. The study highlights the need to prevent and treat early childhood tooth decay as an important part of programmes to prevent child undernutrition as well as NCDs, and to promote children's optimal growth and development during a critical stage of life.

Author Contributions: Conceptualization, B.T., E.P., A.L.; methodology, B.T., E.P., A.L., T.C., S.H.; software, B.T.; validation, B.T.; formal analysis, E.P.R.; investigation, E.P.R., B.T., K.S.-G., S.H., T.C., G.H., E.P., A.L.; resources, B.T., T.C., S.H., A.L., E.P.; data curation, B.T., G.H.; writing-original draft preparation, E.P.R., K.S.-G., B.T.; writing-review and editing, G.H., E.P., A.L., T.C., S.H.; visualization, E.P.R.; supervision, B.T., K.S.-G., G.H.; project administration, G.H., T.C., S.H.; funding acquisition B.T., T.C., S.H., A.L., E.P. All authors have read and agreed to the published version of the manuscript.

Funding: Grant from The Borrow Foundation.

Institutional Review Board Statement: The study was conducted according to the guidelines of the Declaration of Helsinki, and approved by the National Ethics Committee for Health Research, Cambodia (117 NECHR January 2016).

Informed Consent Statement: Informed consent was obtained from all subjects involved in the study.

Data Availability Statement: Restrictions apply to the availability of these data. Data was obtained from the Health and Nutrition Monitoring Study (CAHENMS) in the North East of Cambodia 2016-2019 and are available with the permission of the principal investigator.

Acknowledgments: UNICEF and the Cambodian Oral Health Bureau.

Conflicts of Interest: The authors declare no conflict of interest.

\section{Appendix A. Overview of the CAHENMS Study}

The study monitored health and nutrition indicators for children between birth and 4 years of age and collected individual information about dietary intake, anthropometric measures, cognitive development, the prevalence of diarrhoea and acute respiratory infections as well as information in the household level: SES, handwashing and sanitation. Cases of severe acute malnutrition were referred to the hospital and cases of moderate malnutrition were treated with Numtrey. The wider study included questions about water and hand sanitation, stool, borbor (rice porridge) consumption, and Oral-Health-Related Quality of Life which, were not analysed as part of the present investigation. 
Baseline data were collected in December 2015 and the enrolled children were followed until June 2019; the 6th follow-up (FU6). Data collection and follow-ups were performed every month and every two months after FU5 (Figure A1).

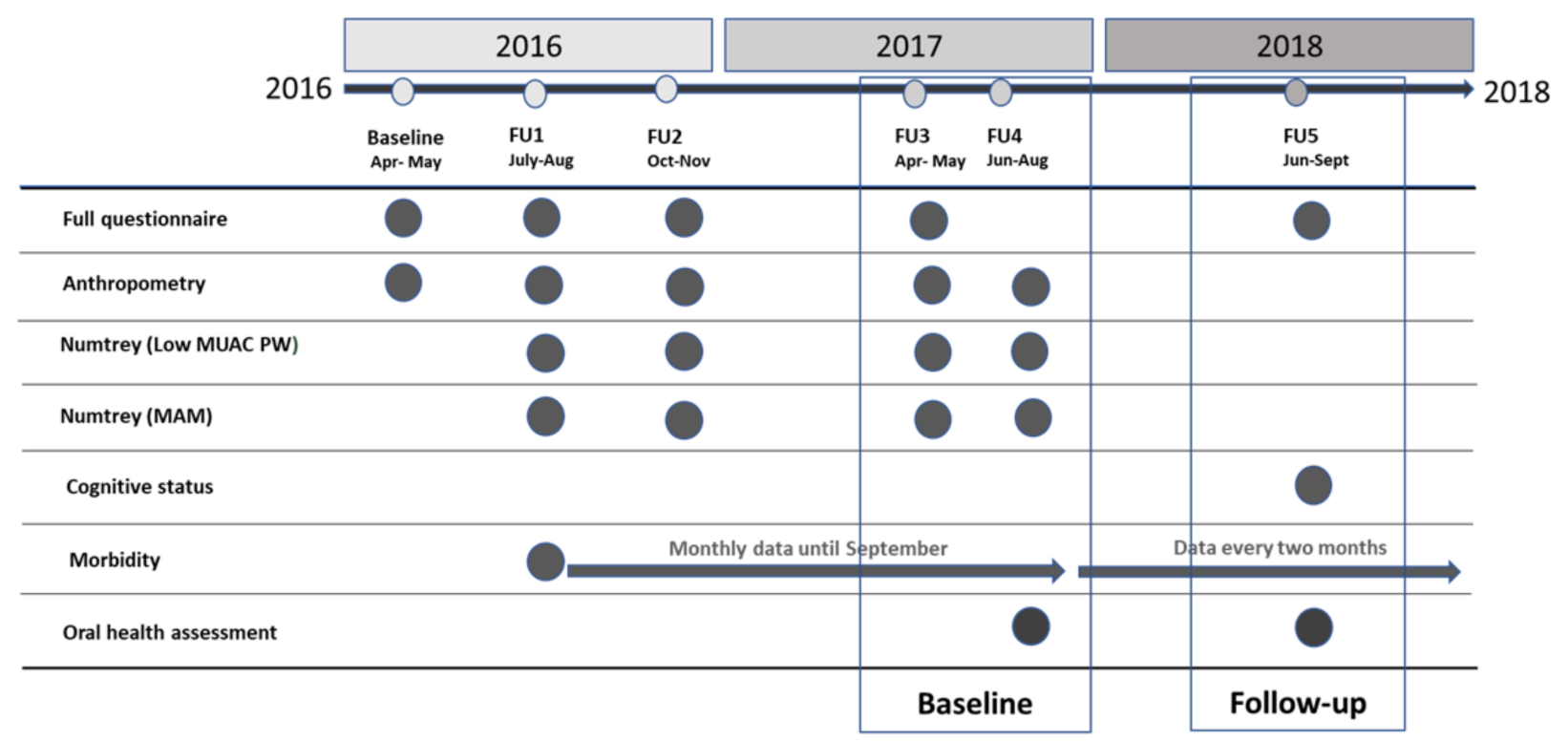

Figure A1. Overview of the CAHENMS study.

The present study used data from follow-up 3 (FU3), follow-up 4 (FU4), and follow-up 5 (FU5), as well as data from children who received an oral examination at CAHENMS FU4 and who were followed a year later at FU5. Parents/caregivers of children who were identified as having carious lesions were informed and instructed to seek further care through a dentist of their choice or through the primary healthcare setting. For the purpose of this analysis, FU3 and FU4 occurring 3 months apart in 2017 were merged as "Baseline"; and FU5 occurring approximately one year later in 2018 was considered as "Follow-up" (FU).

\section{Appendix B. Attrition Analysis}

Table A1 presents the attrition sociodemographic profile of the original sample with 2305 children. There was a statistically significant variation in attrition among age group and province between followed and not-followed children, whereby older children presented higher attrition rate $\left(p<0.001 ; \chi^{2}\right.$ test), although the age group included in the study sample, younger than 23.9 months at baseline, presented similar attrition rates (7-9\%). The province of Ratanakiri presented a higher proportion of children who were lost to follow-up (11.1\% vs. $7.0-7.2 \%, p=0.003 ; \chi^{2}$ test).

Table A1. Attrition analysis of the original sample with sociodemographic profile.

\begin{tabular}{|c|c|c|c|c|}
\hline & $\begin{array}{c}\text { Followed } \\
N(\text { Row \%) }\end{array}$ & $\begin{array}{c}\text { Lost } \\
N(\text { Row \%) }\end{array}$ & $\begin{array}{c}\text { Total } \\
N(\text { Column \%) }\end{array}$ & $p$-Value ${ }^{1}$ \\
\hline & 2107 (91.4) & $198(8.6)$ & 2305 (100.0) & \\
\hline Gender & & & & 0.512 \\
\hline Male & 1057 (91.4) & $99(8.6)$ & $1156(50.2)$ & \\
\hline Female & 1050 (91.4) & $99(8.6)$ & 1149 (49.8) & \\
\hline Age group & & & & $<0.001$ \\
\hline$<6$ months & 143 (92.3) & $12(7.7)$ & $155(6.7)$ & \\
\hline 6-11 months & $280(92.7)$ & $22(7.3)$ & 302 (13.1) & \\
\hline $12-17$ months & 441 (91.5) & $41(8.5)$ & 482 (20.9) & \\
\hline
\end{tabular}


Table A1. Cont.

\begin{tabular}{ccccc}
\hline & $\begin{array}{c}\text { Followed } \\
N \text { (Row \%) }\end{array}$ & $\begin{array}{c}\text { Lost } \\
\text { N (Row \%) }\end{array}$ & $\begin{array}{c}\text { Total } \\
\text { N (Column \%) }\end{array}$ & $p$-Value $\mathbf{1}^{-}$ \\
\hline 18-23 months & $451(91.3)$ & $43(8.7)$ & $494(21.4)$ & \\
24-29 months & $454(95.4)$ & $22(4.6)$ & $476(20.7)$ & \\
30-35 months & $288(90.0)$ & $32(10.0)$ & $320(13.9)$ & \\
36-41 months & $50(65.8)$ & $26(34.2)$ & $76(3.3)$ & \multirow{2}{*}{0.003} \\
Province & & & & \\
Phnom Penh & $493(92.8)$ & $38(7.2)$ & $531(23.0)$ & \\
Kratie & $840(93.0)$ & $63(7.0)$ & $903(39.2)$ & \\
Ratanakiri & $774(88.9)$ & $97(11.1)$ & $871(37.8)$ & \\
SES & & & & \\
Lowest & $347(90.6)$ & $36(9.4)$ & $383(17.4)$ & \\
Low & $528(92.1)$ & $45(7.9)$ & $573(26.1)$ & \\
Medium & $445(89.2)$ & $54(10.8)$ & $499(22.7)$ & \\
High & $352(92.6)$ & $28(7.4)$ & $380(17.3)$ & \\
Highest & $333(92.5)$ & $27(7.5)$ & $260(16.4) 260$ & \\
& & & $260(16.4)$ & \\
\hline
\end{tabular}

${ }^{1} \chi^{2}$ test for differences among groups within the same column.

\section{References}

1. UNICEF; WHO; World Bank Group Joint. Malnutrition in Children; UNICEF: New York, NY, USA, 2020. Available online: https:/ / data.unicef.org/topic/nutrition/malnutrition/ (accessed on 29 August 2020).

2. Roser, M.; Ritchie, H. Hunger and Undernourishment; Our World Data: Oxford, UK, 2013. Available online: https:/ / ourworldindata. org/hunger-and-undernourishment (accessed on 17 February 2020).

3. Roser, M.; Ritchie, H. Obesity; Our World Data: Oxford, UK, 2017. Available online: https://ourworldindata.org/obesity (accessed on 30 August 2020).

4. Bleich, S.N.; Vercammen, K.A. The negative impact of sugar-sweetened beverages on children's health: An update of the literature. BMC Obes. 2018, 5, 1-27. [CrossRef]

5. Featherstone, J.D.B. Caries prevention and reversal based on the caries balance. Pediatr. Dent. 2006, 28, 128-132.

6. Sheiham, A.; James, W. Diet and Dental Caries. J. Dent. Res. 2015, 94, 1341-1347. [CrossRef]

7. World Health Organization. Ending Childhood Dental Caries; World Health Organization: Geneva, Switzerland, 2019.

8. FDI World Dental Federation. Facts, Figures and Stats; FDI World Dental Federation: Geneva, Switzerland, 25 April 2019. Available online: https: / / www.fdiworlddental.org/oral-health/ask-the-dentist/facts-figures-and-stats (accessed on 26 June 2020).

9. Anil, S.; Anand, P.S. Early childhood caries: Prevalence, risk factors, and prevention. Front. Pediatr. 2017, 5, 157. [CrossRef] [PubMed]

10. Lagerweij, M.D.; Van Loveren, C. Declining caries trends: Are we satisfied? Curr. Oral Heath. Rep. 2015, 2, 212-217. [CrossRef] [PubMed]

11. Verlinden, D.A.; Reijneveld, S.A.; Lanting, C.I.; Van Wouwe, J.P.; Schuller, A.A. Socio-economic inequality in oral health in childhood to young adulthood, despite full dental coverage. Eur. J. Oral Sci. 2019, 127, 248-253. [CrossRef] [PubMed]

12. Alkarimi, H.A.; Watt, R.G.; Pikhart, H.; Jawadi, A.H.; Sheiham, A.; Tsakos, G. Impact of treating dental caries on schoolchildren's anthropometric, dental, satisfaction and appetite outcomes: A randomized controlled trial. BMC Public Health 2012, 12, 706. [CrossRef]

13. Khanh, L.N.; Ivey, S.L.; Sokal-Gutierrez, K.; Barkan, H.; Ngo, K.M.; Hoang, H.T.; Vuong, I.; Thai, N. Early childhood caries, mouth pain, and nutritional threats in vietnam. Am. J. Public Health 2015, 105, 2510-2517. [CrossRef]

14. So, M.; Ellenikiotis, Y.A.; Husby, H.M.; Paz, C.L.; Seymour, B.; Sokal-Gutierrez, K. Early childhood dental caries, mouth pain, and malnutrition in the ecuadorian amazon region. Int. J. Environ. Res. Public Health 2017, 14, 550. [CrossRef]

15. World Health Organization. Essential Nutrition Actions: Improving Maternal, Newborn, Infant and Young Child Health and Nutrition; WTO: Geneva, Switzerland, 2013.

16. Cambodia Centrifugal Sugar Human Domestic Consumption by Year (1000 MT). 2020. Available online: https://www. indexmundi.com/agriculture/?country=kh\&commodity=centrifugal-sugar\&graph=human-domestic-consumption (accessed on 20 August 2020).

17. World Health Organization. Who's Oral Health Country/Area Profile Programme (Capp) Database; WTO: Geneva, Switzerland, 2013. Available online: https:/ / capp.mau.se/country-areas/ (accessed on 30 August 2020).

18. National Institute of Statistics. Cambodia Health and Demographic Survey 2014; ICF Macro: Calverton, MD, USA, 2014.

19. Duangthip, D.; Gao, S.S.; Lo, E.C.M.; Chu, C.H. Early childhood caries among 5- to 6-year-old children in Southeast Asia. Int. Dent. J. 2017, 67, 98-106. [CrossRef] 
20. Chher, T.; Turton, B.J.; Hak, S.; Beltran, E.; Courtel, F.; Durward, C.; Hobdell, M. Dental caries experience in Cambodia: Findings from the 2011 Cambodia national oral health survey. J. Int. Oral Health 2016, 8, 1.

21. Oral Health Office; Preventive Medicine Department Cambodia. Cambodia National Oral Health Survey 2011; Preventive Medicine Department Cambodia: Phnom Penh, Cambodia, 2011.

22. Turton, B.; Chher, T.; Sabbah, W.; Durward, C.; Sithan, H.; Laillou, A. Epidemiological survey of early childhood caries in Cambodia. BMC Oral Health 2019, 19, 107. [CrossRef] [PubMed]

23. Victora, C.G.; De Onis, M.; Hallal, P.C.; Blössner, M.; Shrimpton, R. Worldwide timing of growth faltering: Revisiting implications for interventions. Pediatrics 2010, 125, e473-e480. [CrossRef] [PubMed]

24. De Onis, M.; Branca, F. Childhood stunting: A global perspective. Matern. Child Nutr. 2016, 12, 12-26. [CrossRef]

25. Yang, Y.Y.; Kaddu, G.; Ngendahimana, D.; Barkoukis, H.; Freedman, D.; Lubaale, Y.A.; Mupere, E.; Bakaki, P.M. Trends and determinants of stunting among under-5s: Evidence from the 1995, 2001, 2006 and 2011 Uganda Demographic and Health Surveys. Public Health Nutr. 2018, 21, 2915-2928. [CrossRef]

26. Sultana, P.; Rahman, M.; Akter, J. Correlates of stunting among under-five children in Bangladesh: A multilevel approach. BMC Nutr. 2019, 5, 1-12. [CrossRef]

27. Ikeda, N.; Irie, Y.; Shibuya, K. Determinants of reduced child stunting in Cambodia: Analysis of pooled data from three Demographic and Health Surveys. Bull. World Health Organ. 2013, 91, 341-349. [CrossRef]

28. Dewey, R.J.; Arimond, C.M.; Ruel, M.T. Developing and Validating Simple Indicators of Complementary Food Intake and Nutri-Ent Density for Breastfed Children in Developing Countries; Academy for Educational Development: Washington, DC, USA, 2006. Available online: https://pdf.usaid.gov/pdf_docs/Pnadj223.pdf (accessed on 20 October 2020).

29. Hondru, G.; Laillou, A.; Wieringa, F.T.; Poirot, E.; Berger, J.; Christensen, D.; Roos, N. Age-appropriate feeding practices in cambodia and the possible influence on the growth of the children: A longitudinal study. Nutrients 2019, 12, 12. [CrossRef]

30. Som, S.V.; Van Der Hoeven, M.; Laillou, A.; Poirot, E.; Chan, T.; Polman, K.; Ponce, M.C.; Wieringa, F.T. Adherence to child feeding practices and child growth: A retrospective cohort analysis in cambodia. Nutrients 2020, 13, 137. [CrossRef]

31. Filmer, D.; Pritchett, L.H. Estimating wealth effects without expenditure data-or tears: An application to educational enrollments in states of India. Demography 2001, 38, 115-132. [CrossRef]

32. World Health Organization. Who Child Growth Standards: Training Course on Child Growth Assessment; WTO: Geneva, Switzerland, 2008.

33. Petersen, P.E.; Baez, R. World Health Organization. Oral Health Surveys: Basic Methods, 5th ed; World Health Organization: Paris, France, 2013.

34. World Health Organisation. Data Sources and Inclusion Criteria; WHO: Geneva, Switzerland, 2020. Available online: https: //www.who.int/nutrition/databases/infantfeeding/data_source_inclusion_criteria/en/ (accessed on 10 October 2020).

35. World Health Organization. 2010. Indicators for Assessing Infant and Young Child Feeding Practices: Part 2: Measurement; WTO: Geneva, Switzerland, 2020.

36. National Nutrition Program. Nutrition Handbook for the Family; FAO: Rome, Italy, 2011.

37. Bratthall, D. Introducing the significant caries index together with a proposal for a new global oral health goal for 12-year-olds. Int. Dent. J. 2000, 50, 378-384. [CrossRef] [PubMed]

38. Benzian, H.; Monse, B.; Heinrich-Weltzien, R.; Hobdell, M.; Mulder, J.; Helderman, W.V.P. Untreated severe dental decay: A neglected determinant of low body mass index in 12-year-old Filipino children. BMC Public Health 2011, 11, 558. [CrossRef] [PubMed]

39. Shen, A.; Bernabé, E.; Sabbah, W. The bidirectional relationship between weight, height and dental caries among preschool children in China. PLoS ONE 2019, 14, e0216227. [CrossRef] [PubMed]

40. Mishu, M.P.; Hobdell, M.; Khan, M.H.; Hubbard, R.M.; Sabbah, W. Relationship between Untreated dental caries and weight and height of 6- to 12-year-old primary school children in Bangladesh. Int. J. Dent. 2013, 2013, 1-5. [CrossRef] [PubMed]

41. Tsang, C.; Sokal-Gutierrez, K.; Patel, P.; Lewis, B.; Huang, D.; Ronsin, K.; Baral, A.; Bhatta, A.; Khadka, N.; Barkan, H.; et al. Early childhood oral health and nutrition in urban and rural Nepal. Int. J. Environ. Res. Public Health 2019, 16, 2456. [CrossRef]

42. Alshihri, A.A.; Rogers, H.J.; Alqahtani, M.A.; Aldossary, M.S. Association between dental caries and obesity in children and young people: A narrative review. Int. J. Dent. 2019, 2019, 1-8. [CrossRef]

43. Chen, D.; Zhi, Q.; Zhou, Y.; Tao, Y.; Wu, L.; Lin, H.-C. Association between dental caries and BMI in children: A systematic review and meta-analysis. Caries Res. 2018, 52, 230-245. [CrossRef]

44. Hayden, C.; Bowler, J.O.; Chambers, S.; Freeman, R.; Humphris, G.M.; Richards, D.; Cecil, J.E. Obesity and dental caries in children: A systematic review and meta-analysis. Community Dent. Oral Epidemiol. 2013, 41, 289-308. [CrossRef]

45. Hooley, M.; Skouteris, H.; Boganin, C.; Satur, J.; Kilpatrick, N. Body mass index and dental caries in children and adolescents: A systematic review of literature published 2004 to 2011. Syst. Rev. 2012, 1, 57. [CrossRef]

46. Paisi, M.; Kay, E.J.; Bennett, C.; Kaimi, I.; Witton, R.; Nelder, R.; Lapthorne, D. Body mass index and dental caries in young people: A systematic review. BMC Pediatr. 2019, 19, 1-9. [CrossRef]

47. Shivakumar, S.; Shivakumar, G.C.; Srivastava, A. Body mass index and dental caries: A systematic review. Int. J. Clin. Pediatr. Dent. 2018, 11, 228-232. [CrossRef] [PubMed] 
48. Anik, A.I.; Rahman, M.M.; Rahman, M.M.; Tareque, M.I.; Khan, M.N.; Alam, M.M. Double burden of malnutrition at household level: A comparative study among Bangladesh, Nepal, Pakistan, and Myanmar. PLoS ONE 2019, 14, e0221274. [CrossRef] [PubMed]

49. Benefice, E.; Lévi, P.; Banouvong, P. Progressive growth deterioration in a context of nutritional transition: A case study from Vientiane (Lao PDR). Ann. Hum. Biol. 2012, 39, 239-246. [CrossRef] [PubMed]

50. Cordero, M.L.; Cesani, M.F. Nutritional transition in schoolchildren from Tucumán, Argentina: A cross-sectional analysis of nutritional status and body composition. Am. J. Hum. Biol. 2019, 31, e23257. [CrossRef] [PubMed]

51. Ghattas, H.; Acharya, Y.; Jamaluddine, Z.; Assi, M.; El Asmar, K.; Jones, A.D. Child-level double burden of malnutrition in the MENA and LAC regions: Prevalence and social determinants. Matern. Child Nutr. 2020, 16, e12923. [CrossRef]

52. De Mola, C.L.; Quispe, R.; Valle, G.A.; Poterico, J.A. Nutritional Transition in children under five years and women of reproductive age: A 15-years trend analysis in Peru. PLoS ONE 2014, 9, e92550. [CrossRef]

53. Vazquez, C.E.; Cubbin, C. Socioeconomic status and childhood obesity: A review of literature from the past decade to inform intervention research. Curr. Obes. Rep. 2020, 2020,1-9. [CrossRef]

54. World Health Organization. Guideline: Sugars Intake for Adults and Children; WTO: Geneva, Switzerland, 2015.

55. Mühlendahl, K.E.V.; Kromburg, S. A clumsy 3-year-old who liked junk food. Lancet 1996, 348, 1705. [CrossRef]

56. Smith, M.M.; Lifshitz, F. Excess fruit juice consumption as a contributing factor in nonorganic failure to thrive. Pediatrics 1994, 93, 438-443.

57. Peres, A.M.; MacPherson, L.M.D.; Weyant, R.J.; Daly, B.; Venturelli, R.; Mathur, M.R.; Listl, S.; Celeste, R.K.; Guarnizo-Herreño, C.C.; Kearns, C.; et al. Oral diseases: A global public health challenge. Lancet 2019, 394, 249-260. [CrossRef]

58. Bommer, C.; Vollmer, S.; Subramanian, S.V. How socioeconomic status moderates the stunting-age relationship in low-income and middle-income countries. BMJ Glob. Health 2019, 4, e001175. [CrossRef] [PubMed]

59. Gambhir, R.S.; Batth, J.S.; Arora, G.; Anand, S.; Bhardwaj, A.; Kaur, H. Family physicians' knowledge and awareness regarding oral health: A survey. J. Educ. Health Promot. 2019, 8, 45. [CrossRef] [PubMed]

60. Kearns, C.E.; Bero, L.A. Conflicts of interest between the sugary food and beverage industry and dental research organisations: Time for reform. Lancet 2019, 394, 194-196. [CrossRef]

61. Abassi, M.M.; Sassi, S.; El Ati, J.; Ben Gharbia, H.; Delpeuch, F.; Traissac, P. Gender inequalities in diet quality and their socioeconomic patterning in a nutrition transition context in the Middle East and North Africa: A cross-sectional study in Tunisia. Nutr. J. 2019, 18, 18. [CrossRef]

62. Richards, E.; Theobald, S.; George, A.; Kim, J.C.; Rudert, C.; Jehan, K.; Tolhurst, R. Going beyond the surface: Gendered intrahousehold bargaining as a social determinant of child health and nutrition in low and middle income countries. Soc. Sci. Med. 2013, 95, 24-33. [CrossRef]

63. Miller, J.E.; Rodgers, Y. Mother's education and children's nutritional status: New evidence from Cambodia. Asian Dev. Rev. 2009, 26, 131-165.

64. Bell, L.K.; Schammer, C.; Devenish, G.; Ha, D.; Thomson, M.W.; Spencer, A.J.; Do, L.G.; Scott, J.A.; Golley, R.K. Dietary patterns and risk of obesity and early childhood caries in australian toddlers: Findings from an australian cohort study. Nutrients 2019, 11, 2828. [CrossRef]

65. Watt, R.G.; Listl, S.; Peres, M.; Heilmann, A. Social Inequalities in Oral Health: From Evidence to Action; UCL: London, UK, 2015.

66. Petersen, E.P.; Hunsrisakhun, J.; Thearmontree, A.; Pithpornchaiyakul, S.; Hintao, J.; Jürgensen, N.; Ellwood, R.P. School-based intervention for improving the oral health of children in southern Thailand. Community Dent Health 2015, 32, 44-50.

67. Jürgensen, N.; Petersen, P.E. Promoting oral health of children through schools—results from a WHO global survey. Community Dent Health 2013, 30, 204-218.

68. Phantumvanit, P.; Makino, Y.; Ogawa, H.; Rugg-Gunn, A.; Moynihan, P.; Petersen, P.E.; Evans, R.W.; Feldens, C.A.; Lo, E.; Khoshnevisan, M.H.; et al. WHO global consultation on public health intervention against early childhood caries. Community Dent. Oral Epidemiol. 2018, 46, 280-287. [CrossRef]

69. World Health Organisation. Exclusive Breastfeeding for Optimal Growth, Development and Health of Infants; WHO: Geneva, Switzerland, 2020. Available online: http:/ / www.who.int/elena/titles/exclusive_breastfeeding/en/ (accessed on 20 August 2020).

70. World Health Organization. Complementary Feeding: Report of The Global Consultation, and Summary of Guiding Principles For Com-Plementary Feeding of The Breastfed Child; WHO: Geneva, Switzerland, 2003. Available online: https://apps.who.int/iris/ handle/10665/42739 (accessed on 20 August 2020).

71. Dominguez, E.I. Baby WASH and the 1000 Days_A Practical Package for Stunting Reduction; Action Against Hunger: Madrid, Spain, 2017. Available online: https://www.actionagainsthunger.org/sites/default/files/publications/2017_BabyWASH_EN.pdf (accessed on 20 August 2020).

72. World Health Organization. Guidance on Ending the Inappropriate Promotion of Foods for Infants and Young Children: Implementa-Tion Manual; WTO: Geneva, Switzerland, 2017.

73. Turton, B.; Durward, C.; Crombie, F.; Sokal-Gutierrez, K.; Soeurn, S.; Manton, D.J. Evaluation of a community-based early childhood caries (ECC) intervention in Cambodia. Community Dent. Oral Epidemiol. 2020. [CrossRef] [PubMed]

74. Petersen, P.E.; Ogawa, H. Prevention of dental caries through the use of fluoride-the WHO approach. Community Dent. Health 2016, 33, 66-68. [PubMed]

75. Yeung, C.A. A systematic review of the efficacy and safety of fluoridation. Evid. Based Dent. 2008, 9, 39-43. [CrossRef] [PubMed] 
76. Wright, J.T.; Tampi, M.; Graham, L.; Estrich, C.; Crall, J.J.; Fontana, M.; Gillette, E.J.; Nový, B.B.; Dhar, V.; Donly, K.; et al. Sealants for preventing and arresting pit-and-fissure occlusal caries in primary and permanent Molars. Pediatr. Dent. 2016, 38, 282-308. [CrossRef] [PubMed]

77. Lenzi, T.L.; Montagner, A.F.; Soares, F.; Rocha, R.D.O. Are topical fluorides effective for treating incipient carious lesions? J. Am. Dent. Assoc. 2016, 147, 84-91.e1. [CrossRef] [PubMed]

78. Duangthip, D.; Chen, K.J.; Gao, S.S.; Lo, E.C.M.; Chu, C.H. Managing early childhood caries with atraumatic restorative treatment and topical silver and fluoride agents. Int. J. Environ. Res. Public Health 2017, 14, 1204. [CrossRef] 